\title{
AOR
}

Selected Papers of \#AolR2019:

The $20^{\text {th }}$ Annual Conference of the Association of Internet Researchers Brisbane, Australia / 2-5 October 2019

\section{TRUST IN FRIENDSHIP: LGBTQ+ YOUNG PEOPLE AND HOOK-UP APP SAFETIES}

\author{
Paul Byron \\ Swinburne University of Technology \\ Kath Albury \\ Swinburne University of Technology \\ Tinonee Pym \\ Swinburne University of Technology \\ Kane Race \\ University of Sydney \\ Anthony McCosker \\ Swinburne University of Technology
}

\section{Background \& Method}

Digital media research commonly explores the use of social media platforms and dating/hook-up apps separately, and this can imply distance between social and sexual communication practices. Exploring how friendships enfold into LGBTQ+ young people's use of dating/hook-up apps, this paper troubles that delineation.

Many dating/hook-up apps allow users to indicate that they're seeking friends, and friendship-seeking in apps for queer men has been discussed (Castañeda 2015; Chan 2017; Fitzpatrick et al. 2015). Few have considered this beyond apps specific to queer men, however, or how existing friendships influence dating/hook-up app use.

In late 2018, we ran workshops with LGBTQ+ young people (18-35 years) about negotiating safety in dating/hook-up apps. Four workshops were held in Sydney and regional New South Wales (2 each), split between users of apps like Grindr or HER (2 each), and involving 23 participants. Discussion of friendship featured in all workshops, mostly relating to four key themes: the safety of having mutual friends with potential

Suggested Citation: Byron, P., Albury, K., Pym, T., Race, K. and McCosker, A. (2019, October 2-5). Trust in friendship: LGBTQ+ Young People and Hook-up App Safeties. Paper presented at AolR 2019: The $20^{\text {th }}$ Annual Conference of the Association of Internet Researchers. Brisbane, Australia: AolR. Retrieved from http://spir.aoir.org. 
dates/hook-ups; making friends through apps; friends' involvement in safety strategies; and friendship support for app-use negotiation.

\section{Mutuals}

The assurance of having mutual friends with potential dates/hook-ups was commonly discussed. The presence of 'mutuals' was typically established through apps such as Tinder, though some participants complained about Tinder not always showing mutual friends. When seeking same-gendered partners on Tinder, having mutuals also helped participants to determine if a match was actually queer.

Participants engaged in a variety of what Kane Race has discussed as 'cross-platform practices' to establish infrastructures of intimacy and support that were capable of preventing unwanted risks and enabling pleasure $(2017,179-80)$, but these were heterogeneous and far from uniform. Some participants noted the value of Instagram links on app profiles as a way to get more information about the person and check for mutuals. One group (female, urban) agreed that there was greater safety in accessing mutuals through Tinder, rather than sharing Instagram accounts which can feel like "an invasion of privacy." Chelsea (regional) suggested that having mutuals is as good as knowing the person: "Normally if I don't know them - mutual friend wise - I'll video call them [before agreeing to meet]."

Elsewhere, participants spoke of finding and meeting people through non-dating platforms such as Instagram, Facebook, and Twitter. Here, mutual friends served a central role in shaping dating practices and cultivating a greater sense of safety.

\section{Making friends}

Several participants indicated that they've used apps to make friends. Max (non-binary, regional), for example, spoke of the value of meeting other trans people on Tinder: "it's pretty fucking scary being out as trans [here]... So being able to meet other trans people on Tinder, to just be friends with them is really good."

Sometimes making friends through dating apps was unintentional, and sometimes these connections were more difficult to define than simply sexual or friend-based, perhaps combining elements of both.

Amy (urban): I had my first hook up on Tinder, which turned into a Facebook and Instagram connection. I say connection, as opposed to friendship, because we've never seen each other again, but we're still friends and she sent me nudes via email.

Amy indicated that she became friends with many Tinder matches. In the other urban group (for users of Grindr and similar apps), Ben expressed sadness about no longer being able to see his friends naked on Tumblr. This movement from discussing friends on dating apps and other social media illustrates how friendships extend into spaces traditionally deemed sexual (e.g. Tumblr naked selfies) through networked media practices. 


\section{Friends on stand-by}

Many participants (particularly female) commonly told their friends about the dates they were going on and sometimes shared their location or had friends 'on stand-by'. Anna (regional) spoke of sport (which she hated) as her secret code with friends: "So if I send them a thing like 'how is the football going?' they know to call me."

Among male participants, safety arrangements were less common, and telling one's friends about a hook-up for precaution often depended on one's 'gut feeling.' Charles (urban) noted that if he felt uneasy about a hook-up he'd still probably go through with it, but would also send a location screenshot to a friend.

Female participants were less inclined than others to meet at someone's home. For them, meeting in public was discussed as normalised, but they also noted that they were less likely than their straight female friends to have their friends on stand-by.

Tessa (urban): My straight girlfriends, when I said I was dating again, they're like, 'oh, when you're going on dates do you need us to text you? Do you need a phone number? Do you need an emergency just in case?' I was like, 'what?'

\section{Friend support for app use}

Several participants spoke of friends helping them set up their profile, including Kat (regional): "it's good when you're just starting out to have somebody to ask those questions." Participants also discussed giving advice to friends on their photos and profiles. Dan (male, regional) stated: "it is a sweeping generalisation but I have to help my straight friends a lot more with their profiles than gay friends." Max (non-binary) agreed, adding: "There's something wrong with straight men's perception of their dating app profiles" and that their photos are "always blurry."

Zoe (regional) pointed out that rather than teaching friends how to use apps - since they're not difficult and most people know the format - support tended to relate more to the different cultures of each app. In the same workshop, Kat discussed getting tips from friends about how long to wait until a connection moves to a meet-up.

\section{Conclusion}

This paper focuses on friendship as an organising force in young people's dating/hookup app practices, and presents the case for greater attention to the porousness of media sites commonly defined as social (e.g. Instagram) or sexual (e.g. Grindr/Tinder). Participants demonstrate that trust in friendship is far greater that trust in the apps themselves, and so this is called upon, at many levels, to negotiate app use. Notably, 'mutuals' offer greater feelings of safety, and the overlap between friendship and sexual connections is apparent in these data, as per further discussion about 'sliding into DMs'. Importantly, participants who were not cisgender men had greater concern for safety, and thus more knowledge on how to negotiate apps (and dating) safely, particularly through their friendships. 


\section{References}

Castañeda, JGM. (2015) Grindring the Self: Young Filipino Gay Men's Exploration of Sexual Identity Through a Geo-Social Networking Application. Philippine Journal of Psychology 48(1), 29-58.

Chan, LS. (2017) Ambivalence in networked intimacy: Observations from gay men using mobile dating apps. New Media \& Society 20(7), 2566-2581.

Fitzpatrick, C., Birnholtz, J., \& Brubaker, J. R. (2015) Social and Personal Disclosure in a Location-Based Real Time Dating App. Paper presented at the 48th Hawaii International Conference on System Sciences (HICSS), Kauai, HI.

Race, K. (2017) The gay science: Intimate experiments with the problem of HIV. New York: Routledge. 